\title{
Toda obra humana puede mejorarse
}

\author{
Every human work can be improved
}

Al proclamar el concepto de Revolución, Fidel, entre otros asertos dijo: "cambiar todo lo que debe ser cambiado."

Para proyectar cambios es conveniente identificar aquellos que nos trajeron a la situación actual y a partir de esas experiencias proponer rectificaciones y transformaciones, también defensa, ratificación y consolidación de lo que "no debe ser cambiando."

En noviembre de 2010 el Ministerio de Salud Pública declaró en el documento "Transformaciones necesarias en el Sistema de Salud Pública" que:

"Nuestro país requiere hoy nuevas decisiones para continuar perfeccionándose, solucionar los problemas actuales, eliminando las causas que le dieron origen desde sus raíces y avanzar con mayores perspectivas y seguridad hacia su desarrollo."

El documento expone el conjunto de decisiones y transformaciones principales que se emprenden de inmediato. En este contexto y partiendo de lo alcanzado, que debe preservarse, es que se realizan las consideraciones que contiene el presente documento.

Recordando el camino andado por la Salud Pública Revolucionaria cubana se hace infinita la lista de acciones, programas y políticas desarrolladas en más de 50 años, a tener en cuenta para estas consideraciones. Se necesita reducir la lista a lo esencial, para que no se dispersen las ideas o se diluyan las acciones.

Siguiendo a Sotolongo, ${ }^{1}$ citado por Espinosa, ${ }^{2}$ se pueden reducir a diez los grandes cambios, los transcendentales, que más influencia han tenido sobre la práctica médica y la formación en los profesionales de salud pública contemporánea cubana:

1. La creación de un sistema nacional único de salud de modelo socialista.

2. La organización de la atención primaria de salud basada en el especialista de medicina general integral (médico) y la enfermera, insertados en el seno de la comunidad, con apoyo institucional de otros profesionales (medicina familiar). 
3. El reconocimiento de la medicina general integral como especialidad médica, con su consiguiente efecto sobre el estatus social y económico de estos nuevos especialistas (los más numerosos del país).

4. El fortalecimiento de los programas de salud dirigidos a la promoción y prevención.

5. La creciente participación popular en las acciones de salud.

6. El reconocimiento de la enfermería como profesión universitaria y el desarrollo consecuente de los planes formadores y de inserción laboral.

7. La introducción de nuevas tecnologías.

8. La creciente participación de otros profesionales en las acciones de salud.

9. La extinción paulatina ya lograda en términos absolutos de la práctica privada.

10. La desaparición del desempleo profesional.

Ya se advirtió que lo enumerado no es todo. Baste señalar la llamada de atención sobre los aspectos económicos que llevan al primer plano de discusión los lineamientos económicos y sociales del VI Congreso del Partido Comunista de Cuba. Por lo relevante del asunto y la extensión y profunda discusión que se ha llevado a cabo sobre el tema, no se trata aquí.

Sobre estas premisas, cómo proyectar el futuro.

Respecto a la creación del sistema nacional único de salud basta insistir en mantener y fortalecer el sistema, enteramente financiado por el estado, de cobertura total y acceso libre de costos directos para la población, tanto en su componente ambulatorio como hospitalario en sus prestaciones, sean de atención primaria o más compleja. Esto es un sistema socialista.

La atención primaria de salud deberá ratificarse como base reorganizativa del sistema y los servicios, con la presencia del médico y la enfermera en la célula más cercana a la población, el consultorio, que deberá ser fortalecido en su capacidad resolutiva sobre todo con la aplicación sistemática y consecuente del método clínico como poderosa tecnología de la medicina y la salud pública. Esto necesitará de la enseñanza del método desde el pregrado a las nuevas generaciones de profesionales.

Debe detenerse, o al menos controlarse cuidadosamente la salida de los médicos especialistas de medicina general integral hacia otras especialidades, que los alejan de la atención primaria y que no permiten su estabilidad en sus consultorios. Esto exige un sistema de estímulos para mantenerse en el ámbito para el que se formaron, con incentivos como fuentes de actualización profesional, acceso a literatura científica, a la docencia e investigación, a la colaboración internacional, y al uso de las modernas tecnologías de comunicación e información. (Internet), y estimulo salarial adecuado.

Es necesario promover el uso intensivo y de calidad mejorada de la promoción de salud, con atención priorizada a lo que propone la prensa no especializada (periódico, revista, radio y televisión), frecuentemente no bien orientada y que fomenta el consumismo de servicios. I gualmente la prevención deberá protegerse, desplegando acciones como se ha logrado en los programas de inmunizaciones y restableciendo fortalecido el trabajo en el terreno (visita domiciliaria) con fines de promoción. El trabajo de la prensa es fundamental para alcanzar una elevada cultura sanitaria, que no es la medicalización de la sociedad. 
La participación popular necesita ser llevada a la genuina participación, esto es, dando poder de decisión, empoderamiento, a la población. Considerar la conveniencia de restablecer la Comisión Nacional de Salud del Pueblo, que condujo exitosas acciones, sobre todo en los años 60 del pasado siglo.

Todavía es necesario apoyar, con las distintas acciones que sea necesario, el desarrollo individual y colectivo de los profesionales y técnicos de enfermería, para que con su más alto desempeño puedan ser más eficientes y eficaces en su multilateral trabajo.

La introducción y desarrollo propio de nuevas tecnologías ha sido una vigorosa política sostenida por décadas. Esto ha fortalecido los servicios y dado al pueblo alto dividendos en la preservación y recuperación de sus salud, pero la exaltación de estas nuevas conquistas de la ciencia ha deslumbrado a un público con cierta cultura científica y a la mayoría de los trabajadores de la salud, en especial a los médicos, con un saldo negativo en el deterioro de la aplicación del método clínico y un aumento en el costo de los servicios. Es necesario mantener la política seguida, pero con una rigurosa y oportuna evaluación científica de lo que se va a introducir con vigilancia de sus resultados y riesgos y sin propaganda exagerada, mejor sin ninguna propaganda en los medios de comunicación masiva.

El conocimiento de los problemas de salud que afectan a la población está cada vez más necesitado de científicos que no son del campo de las ciencias médicas. Es necesaria una comunicación más frecuente y profunda con persona y entidades, de otros sectores que pueden ofrecer conocimientos y soluciones a problemas que escapan al campo de la salud: ingenieros, físicos, matemáticos, sociólogos, antropólogos, economistas... Fortalecer la cooperación con la Academia de Ciencias de Cuba, sociedades científicas de otros sectores y con centros (Universidades, Institutos...) se hace indispensable.

La práctica privada está reemergiendo, con lo que considero una forma de corrupción. Debe perseguirse y sancionarse. Una forma tolerable pueden ser los cuidados personales a crónicos y discapacitados por personal desvinculados de los servicios (jubilados).

El empleo pleno a los profesionales y técnicos formados masivamente por la Revolución debe preservarse. No es ahí donde predominan las plantillas infladas, es en el personal administrativo (burocracia), dirigentes y funcionarios innecesarios, que entorpecen la buena administración generado burocratismo.

Con estas consideraciones hacemos un llamado a pensar, meditar, sobre lo propuesto. Puede aceptarse o no lo dicho aquí. Pueden sugerirse nuevas ideas. Todo esto es también una batalla de ideas. Recuérdese que "nuestro deber es luchar". Por la Revolución, por la Salud Pública Revolucionaria cubana.

Profesor Francisco Rojas Ochoa

\section{REFERENCI AS BI BLI OGRÁFICAS}

1. Sotolongo P. La importancia y necesidad de la reflexión epistemológica en el quehacer y en la formación de los profesionales de la salud. Resultado de Tema de Investigación. La Habana: Universidad de La Habana; 1997.

2. Espinosa AD. La clínica y la medicina interna. Pasado, presente y futuro. La Habana: Editorial Ciencias Médicas; 2011. 